\title{
PARTICLE FLUX MEASUREMENTS WITH PUMP LIMTTERS*
}

\author{
P. Mioduszewski \\ Oak Ridge National Laboratory \\ Oak Ridge, Tennessee 37831
}

\begin{abstract}
The pressure buildup in pump limiters is a function of the incident particle flux, the pump limiter geometry, particle-surface interactions, and, under certain conditions, nonlinear effects due to interactions of the neutral gas with the incoming plasma. At sufficiently low density and for a short pump limiter throat, the pressure observed in the pump limiter chamber is a direct measure of the particle flux at the limiter slot. This paper discusses a simple model for the correlation between particle flux and pump limiter pressure. In addition, some estimates are presented on the therralization of fast neutrals in the pump limiter duct and its implications for "enhanced particle collection."
\end{abstract}

\section{DISCLAIMER}

\begin{abstract}
This report was prepared as an account of work sponsored by an agency of the United States Government. Neither the United States Government nor any agency thereof, nor any of their employees, makes any warranty, express or implied, or assumes any legal liability or responsibility for the accuracy, completeness, or usefulness of any information, apparatus, product, or process disclosed, or represents that its use would not infringe privately owned rights. Reference herein to any specific commercial product, process, or service by trade name, trademark, manufacturer, or otherwise does not necessarily constitute or imply its endorsement, recommendation, or favoring by the United States Government or any agensy thereof. The views and opinions of authors expressed herein do not necessarily state or reflect those of the United States Government or any agency thereof.
\end{abstract}

\footnotetext{
'Research sponsored by the Office of Fusion Energy, U.S. Department of Energy, under Contract No. DE-AC05-84OR21400 with Martin Marietta Energy Systems, Inc.
} 


\section{INTRODUCTION}

The pressure buildup in pump limiters is a function of the particle flux incident on the limiter and deflected into the pumping chamber [1]. Modeling this process is complicated because of the complexity of the involved atomic and surface processes. The plasma particles incident on the neutralizer plate are subject to a variety of surface phenomena such as reflection, implantation, desorption, and sheath effects, which all depend on particle energy, material, temperature, surface state, etc. Neutrals re-emitted from the neutralizer plate encounter a large number of possible interactions with the plasma. Detailed modeling of all processes involved is complicated, and sophisticated numerical codes like DEGAS [2] have been developed to handle these complex interactions. However, the necessary complexity of these codes often obscures the governing physics processes that lead to a particular result. Therefore, it is in many cases useful to study the basic physics processes with global models and simple approximations. Although simple models lack the completeness of sophisticated codes, they can contribute to the understanding of the basic processes.

\section{PARTICLE FLUX AND PRESSURE BUILDUP}

We consider a pump limiter system in equilibrium; that is, particle fluxes and pressures are constant in time. The equilibrium is considered to be flux equilibrium rather than pressure equilibrium, since the plasma pressure outside the limiter is one to two orders of magnitude higher than the measured neutral pressure. We assume the densities to be low enough so that gas-plasma interactions in the limiter throat can be neglected. Furthermore, the system is not being pumped. In this case the incoming particle fiux is neutralized and fills the pump limiter chamber with gas up to a pressure at which the backflow of neutrals equals the incident ion flux. This situation is depicted in Fig. 1. The incident particle flux is $\phi=\phi_{0} \exp [-(x-h) / \lambda]$, with $h$ and $\lambda$ the height of the pumping slot and the decay length of the particle flux, respectively. The fraction $\eta \phi$ of the incident flux is collected in 
the limiter chamber and is balanced by the backflow of neutrais $n_{s} \bar{\nabla}_{s} / 4$ at the slot. The particle balance is then

$$
\eta w \phi_{0} \int_{0}^{h} \exp [-(x-h) / \lambda] d x=2 h w \frac{n_{3} \overline{\mathrm{v}}_{3}}{4}
$$

where the factor of 2 accounts for molecular particles and $w$ is the width of the pump limiter slot.

The neutral flux of molecules at the slot $n_{s} \bar{v}_{s} / 4$ is unknown, but it can be inferred from the measured neutrai pressure in the pump limiter chamber. A pressure measurement (with an ion gauge) is usually a measurement of the neutral density combined with the assumption that the gas is at room temperature using the relation $p=n k T$. This is a prerequisite for our further considerations. For this case, the neutral flux can be determined: $n \overline{\mathrm{v}} / 4=p / \sqrt{2 \pi m k T}$ where $m$ is the molecular mass and $T$ is room temperature. Since there is flux equilibrium inside the pump limiter, the neutral backflow at the throat must be equal to the flux at the gauge $n_{3} \bar{v}_{s}=n \bar{v}_{\text {s }}$ even though $n_{3}$ and $\bar{v}_{s}$ are not known independently. The incident flux $\phi_{0}$ in Eq. (1) can then be expressed as

$$
\phi_{0}=\frac{2 h p}{\eta \int_{0}^{h} \exp [-(h-x) / \lambda] d x \sqrt{2 \pi m k T}} .
$$

To determine $\phi_{0}$, the parameters $\lambda$ and $\eta$ must be known in addition to the measured pressure $f$. If $\lambda$ is unknown, only the integrated ion flux into the slot can be determined. The remaining unknown is the collection probability $\eta$.

\section{COLLECTION PROBABILITY}

The collection probability $\eta$ is that fraction of the incident flux $\phi$ that is scattered into the pump limiter chamber and contributes to the pressure buildup. The fraction $(1-\eta)$ 
escapes from the chamber after the collision at the neutralizer plate. The collection probability is not to be confused with the trapping fraction $\gamma$, which is that part of the incident flux that is removed by pamping and does not return to the system. Without pumping, the trapping fraction is zero.

To estimate the collection probability, we must discuss the particle-surface interaction. Depending on the particle energy, which is determined by the sheath potential, a certain fraction of the incident ion flux is reflected as neutral atoms and the remaining fraction is implanted in the surface. For incident energies of $100 \mathrm{eV}$, typical particle reflection is $\boldsymbol{R}_{\mathrm{c}}$ $=0.5$ and energy reflection $R_{E}=0.3$ [3]. The implanted particles eventually recombine and desorb as thermal molecules from the surface. Upon saturation of the surface $(\sim 4 \times$ $10^{17} \mathrm{~cm}^{-2}$ ), each incident particle produces one returning particle. We assume that reflected particles as well as desorbed particles are emitted with a cosine distribution. With increasing energies, the reflected fraction decreases and the process becomes dominated by desorption. We assume that the plasma density is low enough that the particles reflected or desorbed from the plate do not interact with the plasma.

From Fig. 1 it is obvious that the collection probability is a function of $x$ and the base $b_{1}$ or $b_{2}$, respectively. Elementary geometry yields for the collected flux $\phi_{\text {coll }}=\eta \phi$

$$
\phi_{\text {coll }}=\frac{1}{2} \phi_{0} \int_{0}^{h} \exp [-(h-x) / \lambda]\left[1+\sin \left(\frac{\pi}{4}-\arctan \frac{x}{b-x}\right)\right] d x \text {. }
$$

The integral can be solved by linearization or numerical integration. It can be seen from Fig. 1 that increasing the base $b$ does not enhance the collection probability proportionally because (1) the increased base is the projection of the incremental solid angle and (2) the intensity of the incremental flux decreases proportional to the cosine of the emitted flux distribution.

The collection probability $\eta$ as a function of the normalized pump limiter base $b / h$ is shown in Fig. 2 for the pump limiters of the Impurity Study Experiment (ISX-B) with $h$ $=2.5 \mathrm{~cm}$ and $\lambda=5 \mathrm{~cm}$. In ISX-B, two different pump limiters were installed: one with 
one-sided collection and $b=11 \mathrm{~cm}$ and one with two-sided collection and $b=6 \mathrm{~cm}$. According to Fig. 2, the collection probability is only slightly enhanced, from 0.73 to 0.83 , for the longer base. As a design criterion, we find that for $b / h$ larger than 4 , the collection improvement is minute. The actual pump limiter in ISX-B had a mushroom configuration, and Fig. 1 represents the central cut only. Integration over the cylinder zeometry yields $\|$ $=0.63$ for two-sided collection and $\eta=0.76$ for one-sided collection. According to these considerations, the collection probability is fairly high, which is a consequence of (1) the $45^{\circ}$ angle of the neutralizer plate and (2) the cosine distribution of the emitted particle flux.

\section{EXPERIMENTAL RESULTS}

The simpie model described here should be best suited for low-density ohmic discharges in which plasma effects in the pump limiter throat are expected to be negligible. Figure 3 shows the pressures in the two pump limiter modules in equilibrium at lineaveraged densities of $1 \times 10^{13} \mathrm{~cm}^{-3}$. The one-sided limiter shows higher pressure than the two-sided one. This is mostly a consequence of toroidal asymmetries of the particle flux; that is, the flux on the electron-drift side has been observed to be lower than that on the ion-drift side. Thus, the slot on the electron-drift side represents only a small increase in particle collection, but doubles the loss. The net effect is higher pressure with one-sided collection. This effect is even more pronounced in beam-heated discharges. For the particle flux analysis, we consider only the one-sided pump limiter module.

Figure 3 shows how the limiter pressure foliows the plasma density with about a 20-ms delay, which is the response time of the pump limiter system. At constant density, the pressure is in equilibrium. The $\mathrm{H}_{x x}$ signal at the limiter, which is not shown here, has the same shape as the density. This indicates that pressure and particle flux are proportional to each other. By applying Eq. (2) with $\eta=0.76$ and $\lambda=7 \mathrm{crc}$ (Langmuir probe measurement), we obtain the particle flux at the upper edge of the pump liniter, $\phi_{0}=$ $1.75 \times 10^{18} \mathrm{~cm}^{-2} \cdot \mathrm{s}^{-1}$. We can compare this particle flux deduced from the pressure 
measurement with the flux measured using a Langmuir probe at the pump limiter slot. From Langmuir probe data, one usually assumes $n_{e} v_{\|}=n_{e} \alpha c_{3}$, with $c_{s}$ being the ion sound speed and $\alpha=0.1, \ldots, 1$. Since we do not know $\alpha$, we plot $\phi_{0}$ from Eq. (2) over $n_{0} c_{3}$ with $c_{\mathrm{s}}=\sqrt{2 k T_{\mathrm{e}} / m_{\mathrm{i}}}$ and $n_{\mathrm{e}}, T_{\mathrm{e}}$ from Langmuir probe measurements. Figure 4 shows this plot for ohmic and for beam-heated discharges. The highest fluxes correspond to $\bar{n}_{e}=5 \times$ $10^{13} \mathrm{~cm}^{-3}$ in $\mathrm{OH}$ discharges and $\bar{n}_{e}=3.5 \times 10^{13} \mathrm{~cm}^{-3}$ in NBI discharges. Increasing the density beyond 2.5 in beam-heated discharges showed nonlinear effects for which this model is not applicable. As Fig. 4 shows, fluxes deduced from the pressure are consistent with Langmuir probe data for $\alpha \approx 0.2$. For ohmic discharges, $\alpha$ might be slightly higher. In either case, $\alpha$ seems to be a little lower than the values of 0.3 or 0.4 most commonly used. This could reflect too high an estimate for the collection probability $\eta$, which determines $\phi_{0}$ according to Eq. (2). If $\alpha=0.3$ is assumed, the data are consistent with collection probabilities between 0.5 and 0.63 . There is uncertainty in the assumptions made here about the backscattering at the neutralizer plate, although the maximum possible value of $\eta$ appears to be 0.8 and our best estimate of $\eta=0.65 \pm 0.15$ cannot be too far off. Thus, a simple pressure measurement yields the particle flux in the scrapeoff layer, as was shown for $\mathrm{OH}$ discharges with densities up to $5 \times 10^{13} \mathrm{~cm}^{-3}$ and for NBI discharges up to $3.5 \times 10^{13} \mathrm{~cm}^{-3}$.

The data shown in Figs. 3 and 4 are all without active pumping in the limiter. When the $\mathrm{Zr} / \mathrm{Al}$ getter pump inside the pump limiter chamber was activated, the pressure dropped by a factor of 3.2 at high densities to 4 at low densities. A pressure drop of 3.2 requires the particle removal through pump and slot to be 3.2 times higher than through the slot alone, i.e., $\phi_{\mathrm{p}}+\phi_{\mathrm{s}}=3.2 \phi_{\mathrm{s}}$. Hence, the pump removes $68 \%$ of the collected flux. The trapping fraction $\gamma$ is then $\gamma=0.68 \eta \approx 0.5$; that is, $50 \%$ of the incident flux $\phi$ is removed from the system by pumping. 


\section{PARTICLE TRANSPORT IN THE PUMP LIMITER}

For the global particle balance in the pump limiter, it was assumed that the gas at the pressure gauge was at room temperature. To verify this assumption, we try to estimate the time after which a fast particle is thermalized and the corresponding distance it has traveled. Although an analytical approach which includes all the involved physics processes would be too complicated, reasonable assumptions and simplifications can lead to useful results and provide valuable insight.

The diffusion length is $x=\sqrt{D t}$ with a diffusion coefficient $D=(1 / 4) \lambda \bar{v}$. Assuming a cosine distribution of the emitted particles, the mean free path in a tube of diameter $d$ can be computed to be $\lambda=(\pi d) / 2$, where collisions between particles are negligible. The resulting diffusion coefficient $D=(1 / 8) \pi d \bar{v}$ can be shown to be compatible with the usual formula for the conductance of molecular flow. The average particle velocity $\bar{\nabla}$ changes as a function of the wall collisions, and the diffusion coefficient and the time between collisions change correspondingly. In the interval between collisions $k$ and $(k+1)$, we have $D_{k}$ $=(1 / 4) \lambda \bar{v}_{k}$ and $t_{k}=\lambda / \bar{v}_{k}$. The diffusion length is then

$$
\bar{x}=\left(\sum_{k=1}^{N} D_{k} t_{k}\right)^{1 / 2}=\frac{1}{4} \pi d \sqrt{N},
$$

where $N$ is the number of collisions; that is, the diffusion length is determined by the tube diameter and the number of collisions.

We now estimate the number of collisions and the elapsed time that a fast particle needs for thermalization with the wall. In the first approach, we consider the slowing-down process of monoenergetic particles. The energy reflection coefficient $R_{E}$ ior energies between zero and $100 \mathrm{eV}$ can conveniently be represented as $R_{E}=1-0.082 \ln \left(E / E_{\mathrm{w}}\right)$ (for deuterium on iron) where $E$ is the incident energy and $E_{\mathrm{w}}$ is the energy corresponding to wall temperature. If a wider energy range is to be covered, the analytical expression for $R_{E}$ becomes more complicated [4]. When the incident particle energy corresponds to the 
wall temperature, the energy reflection coefficient becomes unity and the energy transfer to the wall is zero.

The energy of the reflected particle is $E_{\mathrm{r}}=R_{E} E$. After $N$ collisions it is

$$
E_{N}=E_{0} \prod_{k=1}^{N}\left[1-0.082 \ln \left(E_{k-1} / E_{\mathrm{w}}\right)\right]
$$

where $I$ indicates the product for $k=1, \ldots, N$ and $E_{0}$ is the initial particle energy. After the $k$ th collision, the particle has a velocity $v_{k}=\sqrt{2 E_{k} / m}$ and the time between collisions is $t_{k}=\lambda / v_{k}$. After the $n$th collision, the elapsed time is

$$
t_{N}=(\pi d / 2) \sum_{k=1}^{N} 1 / \sqrt{2 E_{k} / m}
$$

The particle energy as a function of the number of collisions is plotted in Fig. 5 with initial particle energies of $E_{0}=25 \mathrm{eV}$ and $100 \mathrm{eV}$. The graph shows that the slowing-down process is fast at the beginning when $R_{E}$ is low and becomes very slow when the particle energy approaches wall temperature and $R_{E}$ approaches unity. The corresponding time required for the slowing-down process is shown in Fig. 6. For $25-\mathrm{eV}$ or $100-\mathrm{eV}$ particles in a 3-cm-diam tube, thermalization time is on the order of $10^{-3} \mathrm{~s}$.

In the second approach, we consider a particle ensemble with a Maxwell-Boltzmann velocity distribution. The average velocity of the distribution is $\bar{v}=\sqrt{8 k T / \pi m}$, and the average energy that a particle carries to the wall is $2 k T$ [5]. The energy transferred to the wall in a collision is $2 k T\left(1-R_{E}\right)$. The energy transferred to the wall, per unit time, must be equal to the energy lost by the particle ensemble:

$$
\dot{E}=-\frac{3}{2} n k \dot{T} \cdot V=A \frac{n \vec{v}}{4} 2 k T\left(1-R_{E}\right)
$$


Substituting $R_{E}=1-0.082 \ln \left(T / T_{w}\right)$, we obtain the differential equation

$$
\frac{d T}{d t}=-C T^{3 / 2} \ln \left(\frac{T}{T_{\mathrm{w}}}\right),
$$

where $C$ contains the constants of Eq. (7). Integration of Eq. (8) yields the elapsed time for the thermalization from the initial temperature $T_{0}$ to the temperature $T_{N}$ :

$$
t_{N}=\frac{1}{C} \int_{T_{0}}^{T_{N}} \frac{d T}{T^{3 / 2} \ln \left(T / T_{w}\right)}
$$

With the substitution $2 z=\ln \left(T / T_{\mathrm{w}}\right)$, the integral can be transformed into an exponential integral, which can be computed by its infinite series or by numerical integration. For deuterium gas in a $3-\mathrm{cm}$-diam circular tube, the constant is $C=2.8 \times 10^{4}$. The temperature as a function of elapsed time is shown in Fig. 6 for the initial temperatures of $100 \mathrm{eV}$ and $25 \mathrm{eV}$. Comparison with the monoenergetic particle solution indicates that the slowingdown functions are similar, but that the thermalization of the Maxwell-Bo'tzmann distribution is somewhat faster at the end. This is a consequence of the higher average energy $2 k T$ per particle transported to the wall by the particle distribution. Neither the number of collisions nor the thermalization time changes much when we compare $25-\mathrm{eV}$ and $100-\mathrm{eV}$ particles. Slowing down from $100 \mathrm{eV}$ to $25 \mathrm{eV}$ takes only two collisions because the energy reflection is low at the high-energy end, and the additional time is negligible since the collision times are short.

Once the number of collisions for thermalization is known, the diffusion length can be estimated with Eq. (4). For particles with the initial kinetic energy of $100 \mathrm{eV}$, the diffusion length for thermalization in a $3-\mathrm{cm}$-diam tube is $x=18 \mathrm{~cm}$ and the elapsed time is on the order of $1 \mathrm{~ms}$. The thermalization point is somewhat arbitrary because the wall temperature is approached asymptotically. 
In the ISX-B pump limiter module, the ion gauge was connected to the pump limiter chamber with a $50 . \mathrm{cm}-$ long, $3-\mathrm{cm}$-diam tube; that is, the tube was about 3 times longer than the thermalization length. Hence, we assume that the gas at the ion gauge was in thermal equilibrium with the wall. The space within the pump limiter chamber was to a large extent filled by the $\mathrm{Zr} / \mathrm{Al}$ getter cartridge such that the mean free path for particles was only on the order of $1-3 \mathrm{~cm}$. Therefore, we assume that the particles were thermalized fairly rapidly even within the pump limiter chamber. Consequently, enhanced pumping due to energetic particles could not be expected.

The estimates of the thermalization length can also provide some insight into the effects of "enhanced conductance." This enhancement can be expected if the thermalization length is longer than the pumping duct. As an example, we consider a 10-cm-diam, 100-cm-long duct. The thermalization length according to Eq. (4) is about $50 \mathrm{~cm}$. Hence, the first half of the duct has enhanced conductance, while the second half has normal thermal conductance. Since the resulting conductance is determined by the smaller value, enhancement cannot be expected for particle energies typical for the plasma edge of fusion devices.

\section{CONCLUSIONS}

Some simple modeling has been presented of the global particle balance and the thermalization in pump limiters. The modeling fits the ISX-B pump limiter data reasonably well. It allows us to deduce the particle flux in the scrapeoff layer from simple pressure measurements. If particle control is not the objective, a smaller probe can be designed for scrapeoff layer studies. Estimates of the thermalization length of fast particles in the pump limiter can be obtained from an energy-dependent reflection coefficient and the slowdown process of either monoenergetic particles or a Maxwell-Boltzmann distribution. The thermalization length is almost independent of the initial particle energy as it is observed in the scrapeoff layer of fusion devices. Improved particle collection due to "enhanced conduction" can only occur in short ducts of sufficiently large diameter. 
REFERENCES

[1] P. Mioduszewski, in Physics of Plasma-Wall Interactions in Controlled Fusion, edited by D. E. Post and R. Behrisch, Plenum, New York, 1986.

[2] D. Heifetz et al., J. Comput. Phys. 46 (1982) 309.

[3] W. Eckstein and H. Verbeek, Max-Planck-Institut für Plasmaphysik Report IPP9/32 (1979).

[4] W. Eckstein and H. Verbeek, p. 20 in Data Compendium for Plasma-Surjace Interactions, special issue of Nucl. Fusion (1984).

[5] A. Roth, Vacuum Technology, North-Holland, Amsterdam, 1976, p. 57. 


\section{FIGURE CAPTIONS}

Fig. 1. Geometry and particle fluxes in the ISX-B pump limiter module.

Fig. 2. Collection probability $\eta=\phi_{\mathrm{coll}} / \phi$ as function of $b / h$, the pump limiter base $b$ normalized to the height $h$.

Fig. 3. Density and gas puff of low-density $\mathrm{OH}$ discharge and corresponding limiter pressures due to (a) single-sided collection and (b) two-sided collection.

Fig. 4. Particle flu $\phi_{0}$ deduced from pressure measurement plotted over $n_{e} c_{3}=$ $(1 / \alpha) n_{\mathrm{e}} v_{\|}$from Langmuir probe measurements.

Fig. 5. Particle energy as a function of the number of collisions with the wall for $E_{0}$ $=100 \mathrm{eV}$ and $25 \mathrm{eV}$.

Fig. 6. Thermalization of a Maxwell-Boltzmann distribution of particles and of monoenergetic particles as function of time. 
ORNL-DWG 86-2331 FED

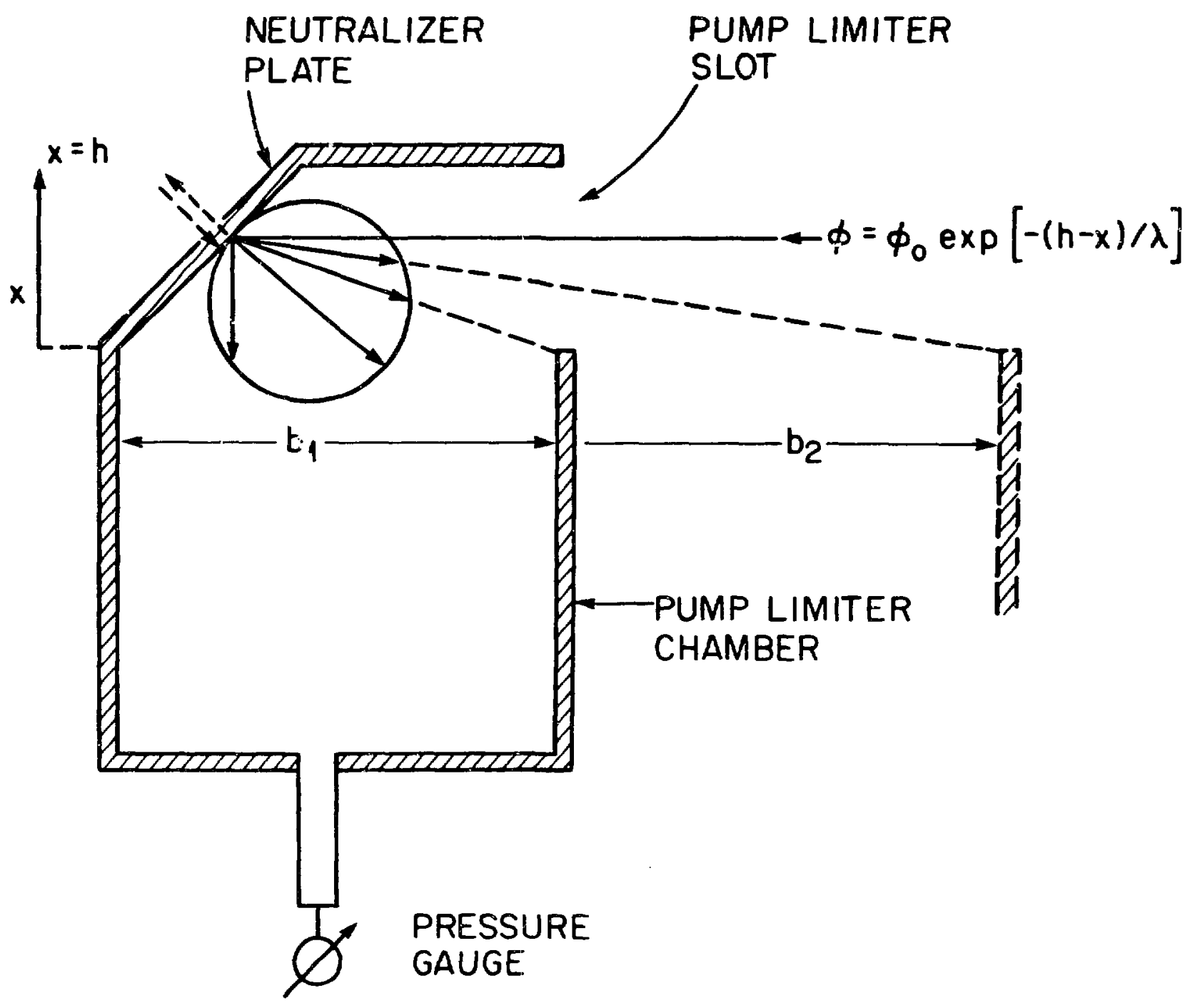




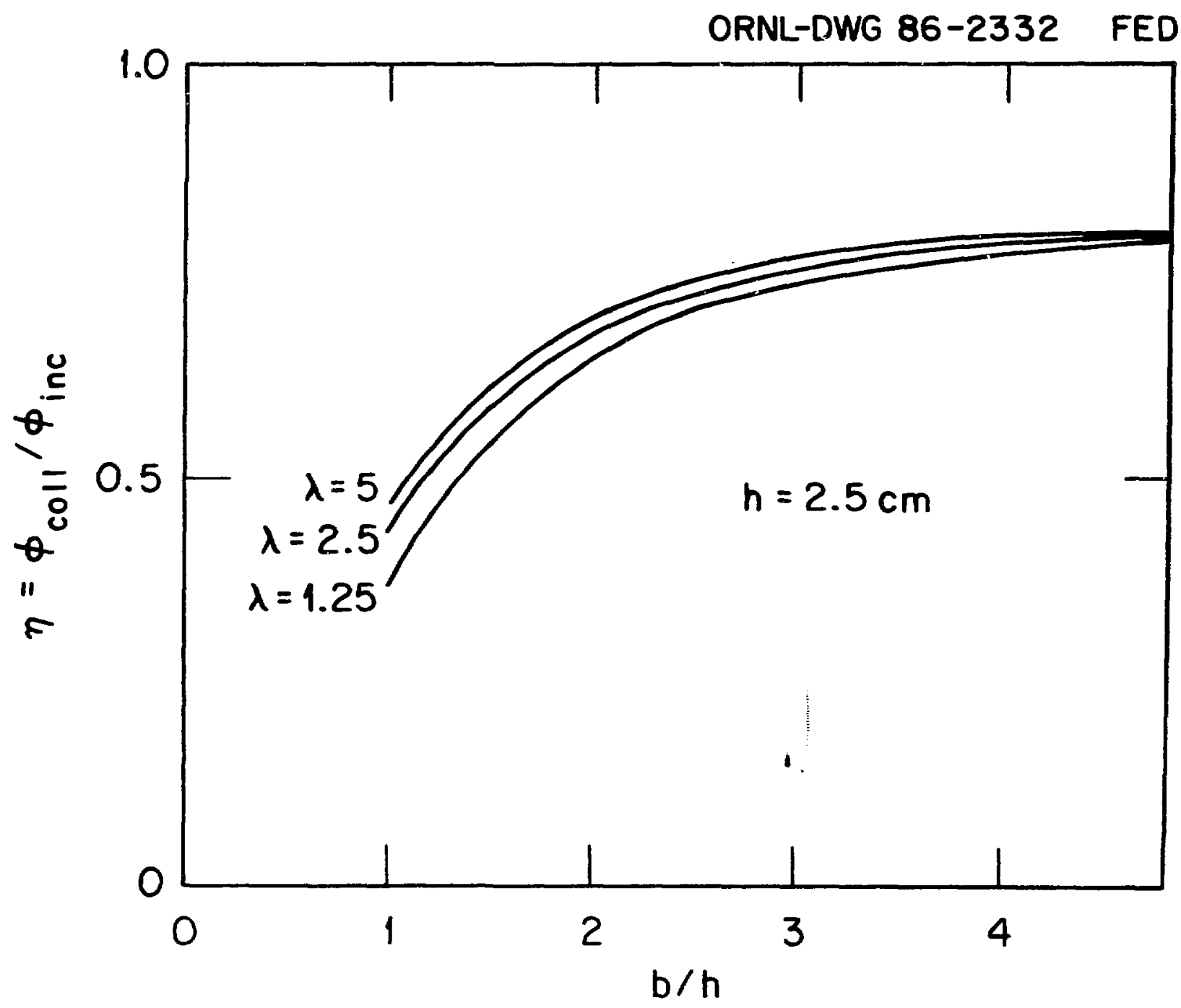


ORNL-DWG 86-2334 FED
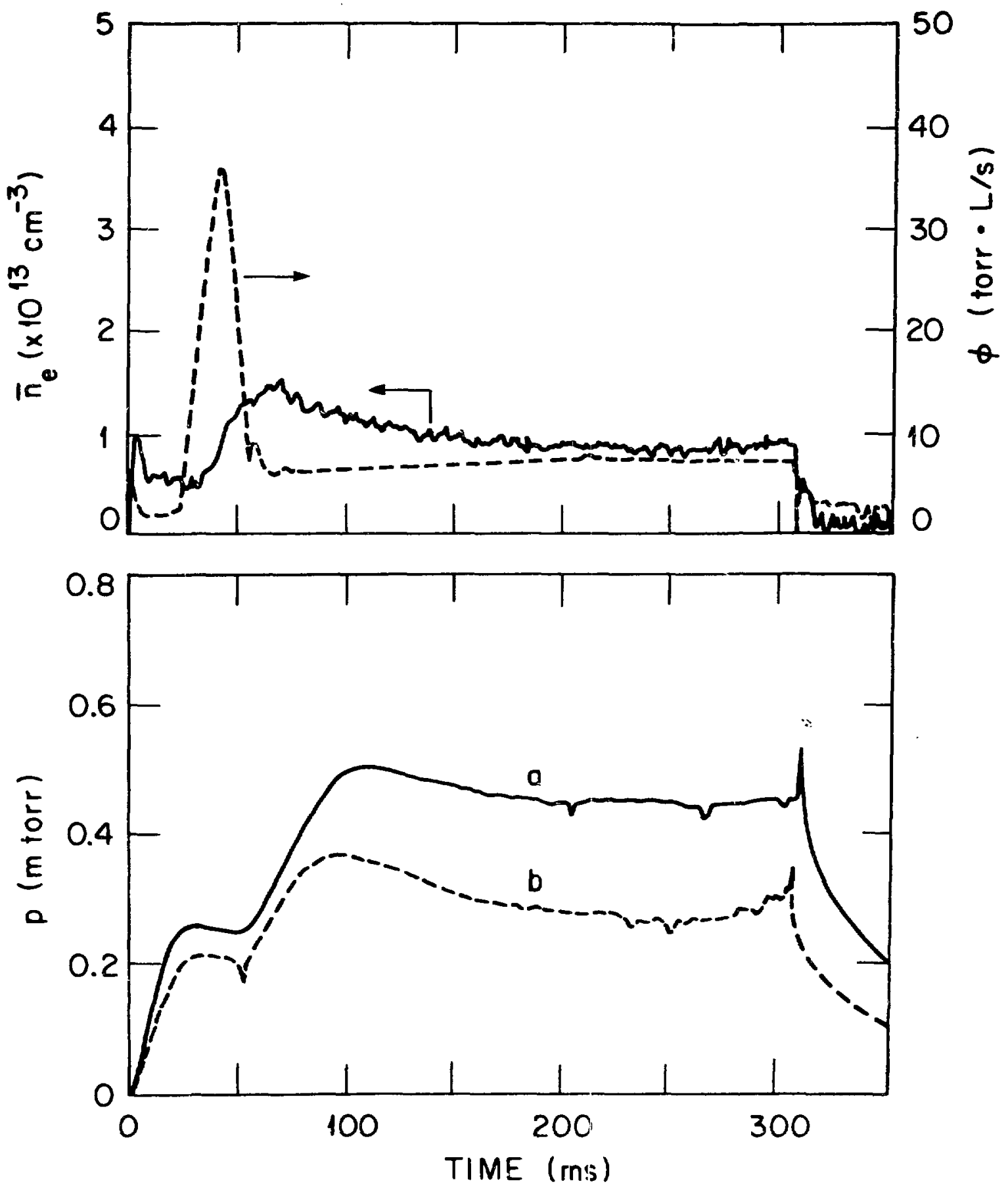


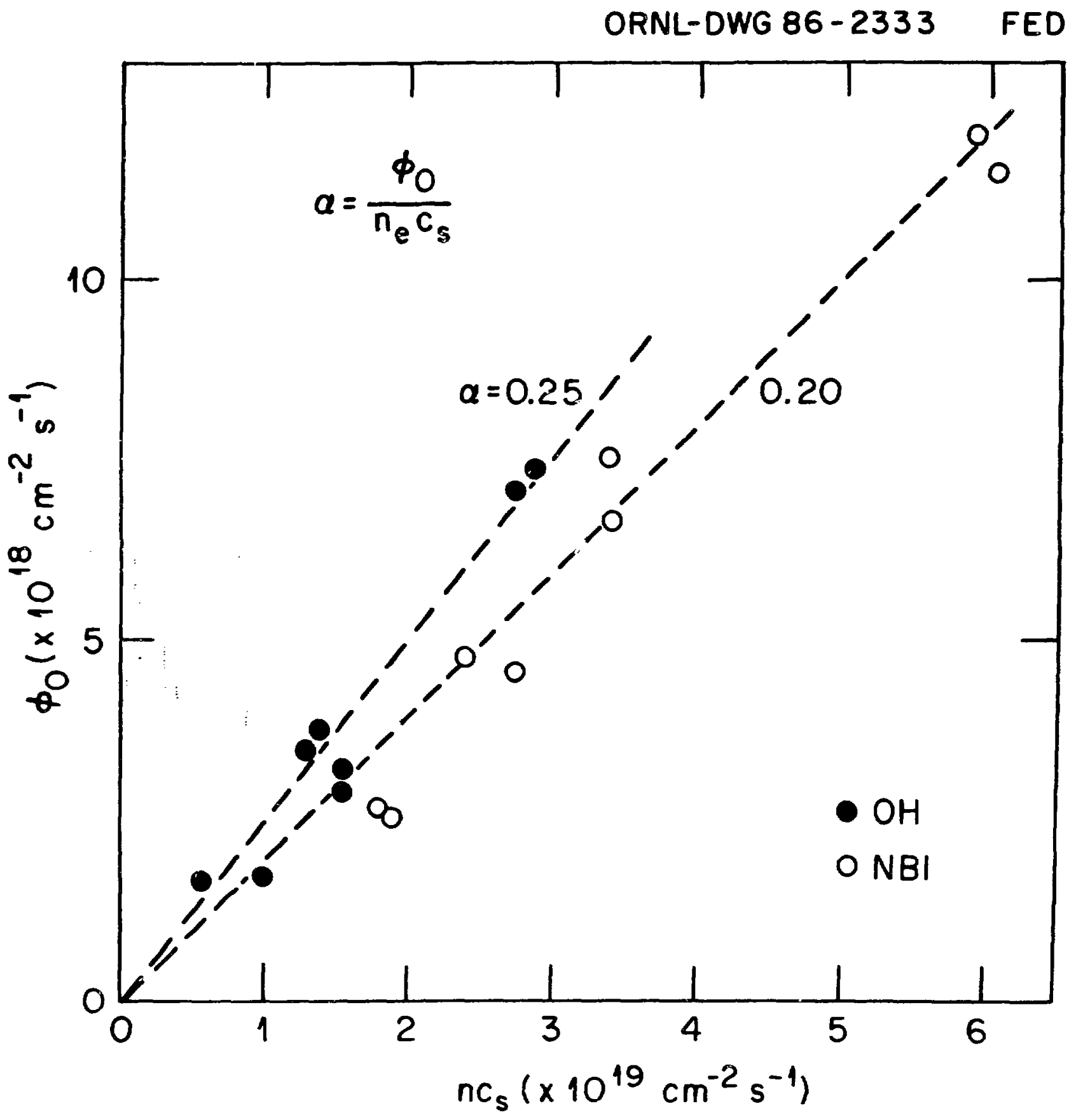




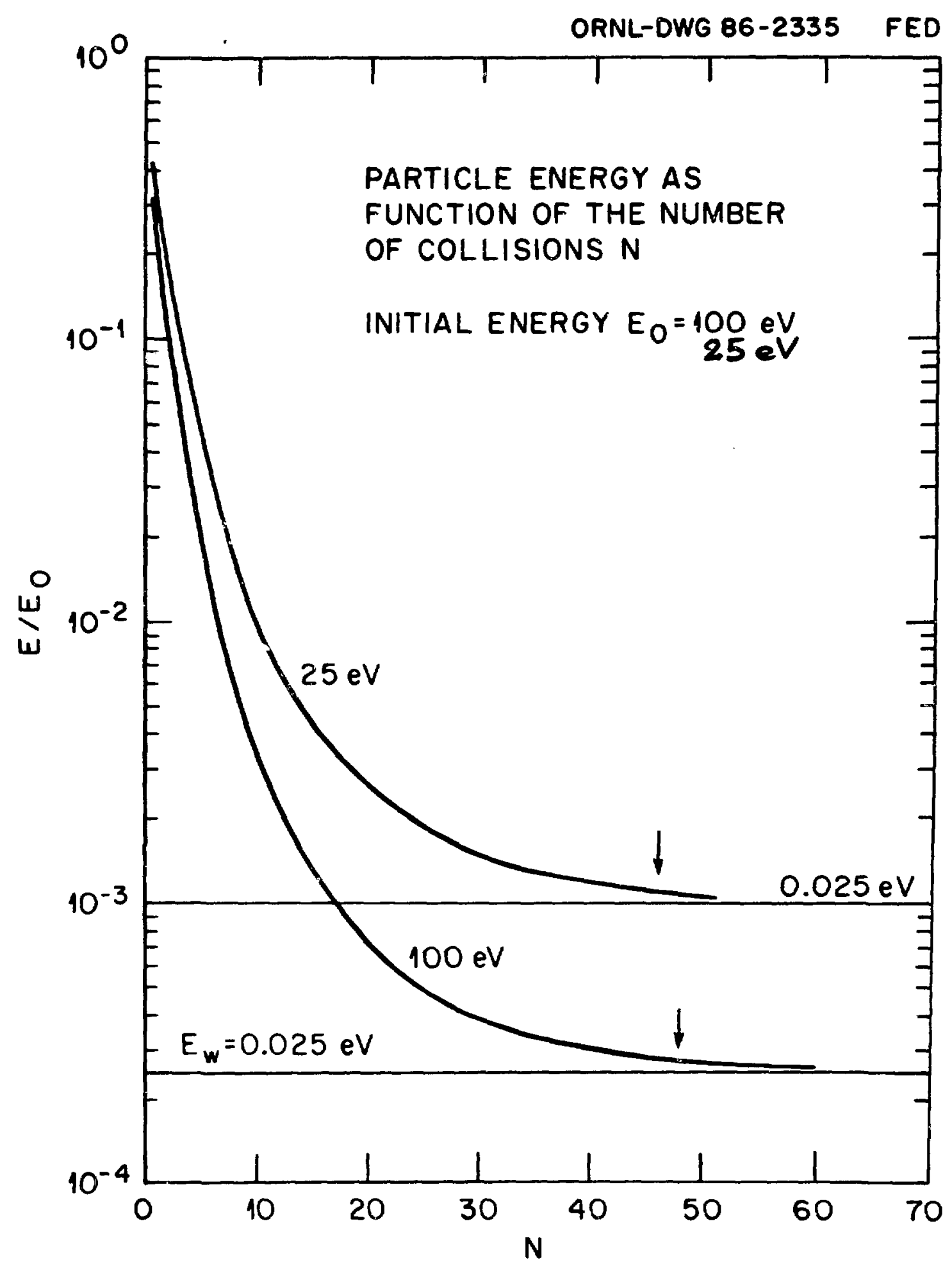




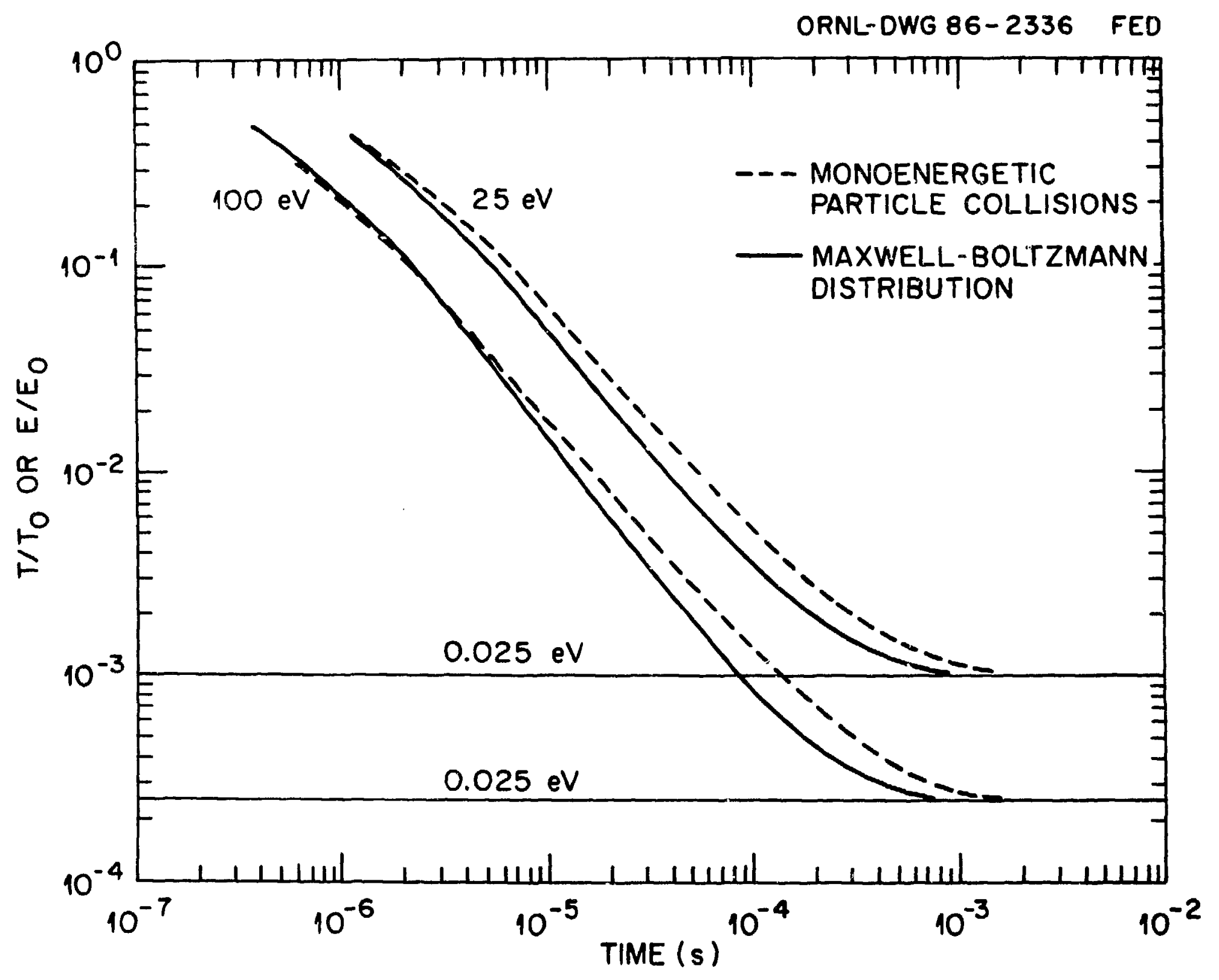

